\title{
Comparison between Edge Detection Techniques
}

\author{
Satbir Kaur \\ Research Scholar \\ CSE \& IT Department \\ Baba Banda Singh Bahadur Engineering College, \\ Fatehgarh Sahib
}

\author{
Ishpreet Singh \\ Assistant Professor \\ CSE \& IT Department \\ Baba Banda Singh Bahadur Engineering College, \\ Fatehgarh Sahib
}

\begin{abstract}
Edge is the foremost feature of the image. Edges can be defined as boundary between regions in an image. Edge detection refers to the process of identifying and locating sharp discontinuities in an image. Edge detection process reduces the amount of data and filters out useless information, while preserving the necessary structural properties in an image. In this paper, the main purpose is to study edge detection process based on different techniques.
\end{abstract}

\section{Keywords}

Edge detection, Sobel, Prewitt, Laplacian of Gaussian, Canny edge detection

\section{INTRODUCTION}

Edge detection is a type of image segmentation techniques which determines the presence of an edge or line in an image and outlines them in an appropriate way [1]. The main purpose of edge detection is to simplify the image data in order to minimize the amount of data to be processed [2]. Generally, an edge is defined as the boundary pixels that connect two separate regions with changing image amplitude attributes. The various edge detection techniques are in the figure 1.1

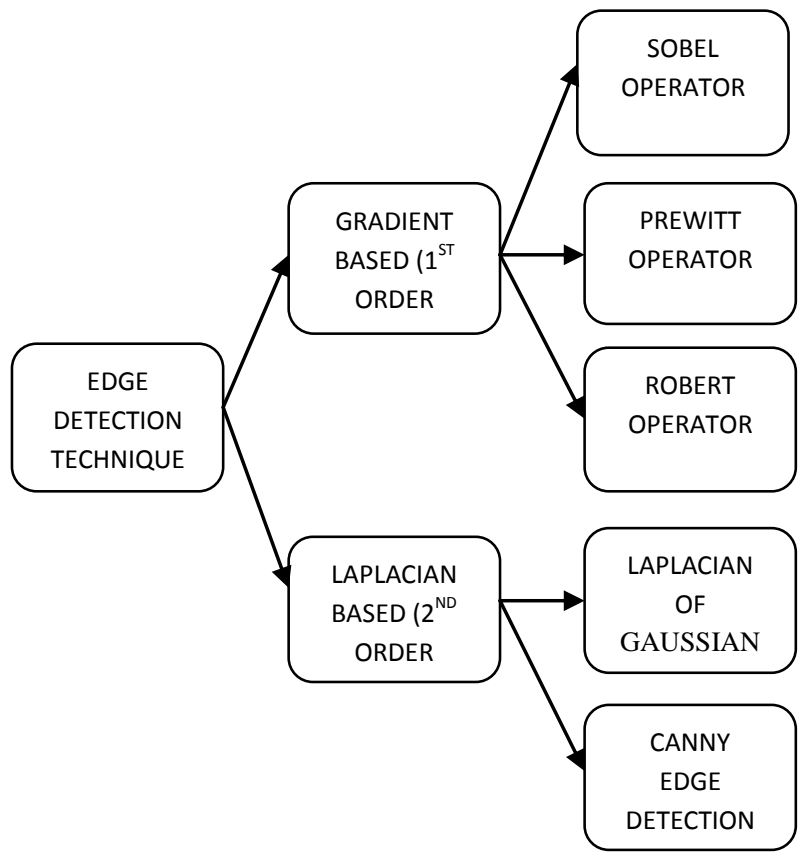

Fig1.1: Various Edge Detection Techniques
The gradient based edge detection method detects the edges by looking for maximum and minimum in the first derivative of the image. First derivatives in image processing are implemented using the magnitude of the gradient [13]. The following are the gradient based techniques

1.1.1. Sobel Operator: It is $3 \times 3$ convolution kernels. One kernel is simply the other rotated by $90^{\circ}$. It is a row edge detector.
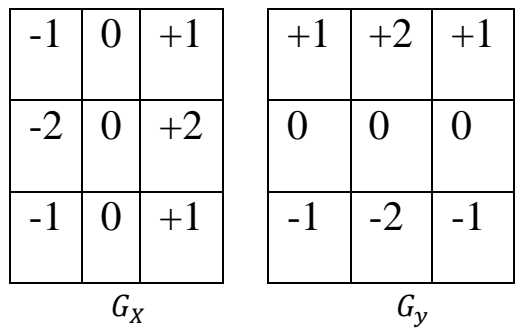

Fig1.2:Sobel mask filter in $\mathrm{X}$ direction and $\mathrm{Y}$ direction

$$
G_{X} \text { And } G_{y} \text { are the common masks used in Sobel Operator }
$$

This figure shows the masks used by Sobel operator. The kernel can be applied separately to input image for obtaining gradient component in each orientation i.e. GX and GY. The magnitude is given by:

$$
|G|=\sqrt{G_{X}^{2}}+G_{y}^{2}
$$

And its approximation is done by:

$$
|\mathrm{G}|=\left|G_{X}\right|+\left|G_{Y}\right|
$$

The orientation of angle is given by:

$$
\theta=\arctan \left(\frac{G_{X}}{G_{Y}}\right)
$$

1.1.2. Prewitt Operator: The prewitt edge detector is an appropriate way to estimate the magnitude and orientation of an edge. The prewitt operator is limited to 8 possible orientations, however most direct orientation estimates are not much more accurate. This gradient based edge detector is estimated in the $3 \times 3$ neighbourhood for 8 directions. All the eight convolution masks are calculated. The convolution mask with the largest module is then selected. The convolution masks of the Prewitt detector are given below: 


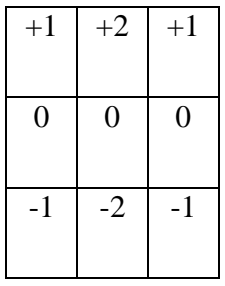

$P_{X}$

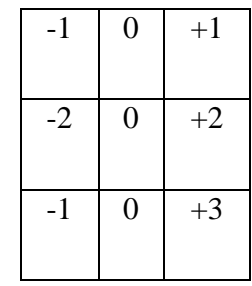

$P_{Y}$

Fig 1.3: Mask filter of Prewitt operator in $X$ and $Y$ direction

\section{Algorithm:}

Input: A Sample Image.

Output: Detected Edges.

Step 1: Accept the input image.

Step 2: Apply mask Gx, Gy to the input image.

Step 3: Apply prewitt edge detection algorithm and the gradient.

Step 4: Masks manipulation of Gx, Gy separately on the input image.

Step 5: Results combined to find the absolute magnitude of the gradient.

Step 6: The absolute magnitude is the output edges.

1.1.3. Robert's cross Operator: This is somewhat similar to Sobel and Prewitt Operator. It is a 2-D spatial gradient measurement of an image. The pixel value represents the absolute magnitude of spatial gradient of input at that point. Operator has $2 \times 2$ convolution kernel. Kernel responds to maximize edges running at 450 to each pixel grid. For each of the perpendicular orientations there is one corresponding kernel.

The magnitude is given by the same formula as of the Sobel but the orientation of the angle is given by:

$$
\theta=\arctan \left(\frac{G_{X}}{G_{Y}}\right)-\frac{3 \pi}{4}
$$

1.2: Laplacian Based Technique: The Laplacian based edge detection method searches for the zero crossing in the second derivative of the image to the edges. The edge has one dimensional shape of the ramp and calculating the derivative of the image can highlight it's location. So the laplacian based edge detection techniques are as follows:

1.2.1. Laplacian of Gaussian (LoG): It was proposed by Marr (1982). The LoG of an image $f(x, y)$ is a second derivative defined as

$$
\nabla^{2} f=\frac{\partial^{2} f}{\partial x^{2}}+\frac{\partial^{2} f}{\partial y^{2}}
$$

It first smoothes the image and then computes the Laplacian. This yields in double edge image; hence for finding the edge the zero crossing between the double edges is taken.

The Laplacian of an image with the pixel intensity value $\mathrm{L}(\mathrm{x}, \mathrm{y})$ is given by:

$$
L(x, y)=\frac{\partial^{2} I}{\partial x^{2}}+\frac{\partial^{2} I}{\partial y^{2}}
$$

The commonly used discrete approximations to Laplacian filter are:
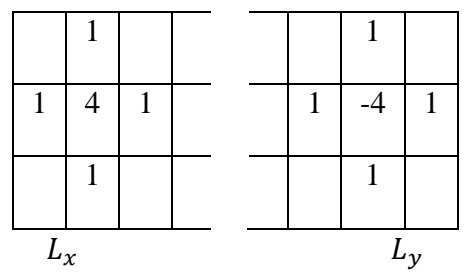

Fig1.4: Laplacian filter in $\mathrm{X}$ direction and $\mathrm{Y}$ direction

The Gaussian filtering is combined with Laplacian to break down the image where the intensity varies to detect the edges effectively [4].

\subsubsection{Canny Edge Detection:}

Canny edge detector is one of the most commonly used image processing tools. It detects edges in a very robust manner. Unlike Roberts Cross and Sobel, the canny operation is not very susceptible to noise. It takes less time than Roberts cross. It is one of the most important methods to find the edges by separating noise from input image. The algorithm is adaptable to various environments. It is a better method because it extracts the features in an image without disturbing its features. There are certain criteria to improve current methods of edge detection. The first and most obvious is low error rate. It is important that edges occurring in images should not be missed. The second criterion is that the edge points be well localized i.e. the distance between the edge pixels as found by the detector and the actual edge should be minimum. A third criterion is to have only one response to a single edge [5].Canny edge detector have advanced algorithm derived from the previous work of Marr and Hildreth. It is an optimal edge detection technique as provide good detection, clear response and good localization. It is widely used in current image processing techniques with further improvements.

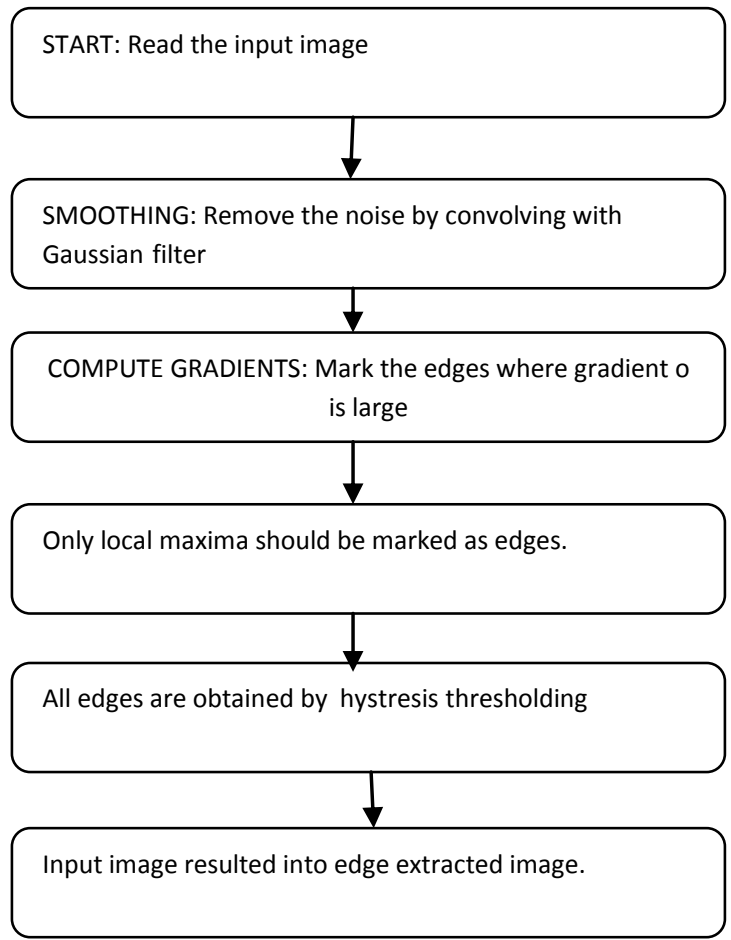

Fig 1.5: Flow chart of canny edge detection 


\section{3: Applications of edge detection:}

The main applications of edge detection techniques are in the process of image segmentation and object detection. Edge maps help in representing faces as a single unit. An edge detection system has wide variety of applications, as shown in below figure 1.3:

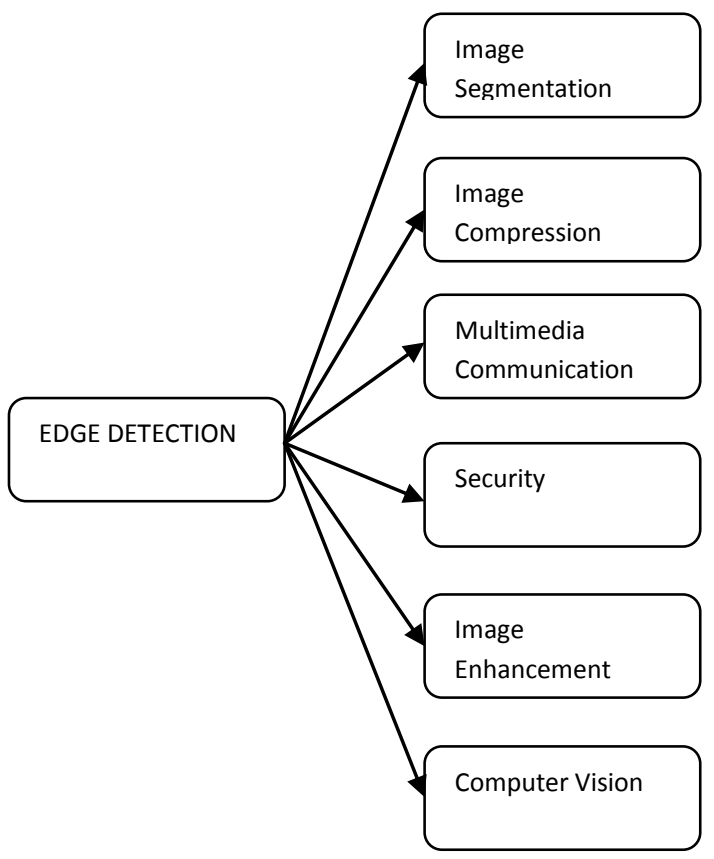

Fig1. 3: Applications of edge detection

\section{4: Image Segmentation:}

Segmentation is a process of distinguishing objects from the background. Hence, Image segmentation is distinguishing or partitioning the image from its background. The four main approaches used for image segmentation are: threshold techniques, edge detection techniques, region-based techniques, and connectivity preserving relaxation methods. Most widely and important amongst these four techniques is "edge detection". The level of the subdivision has to stop when the object or image of interest have been partitioned. Picking up an appropriate technique for "good" segmentation is a challenging task. Edge being such an essential part in an image, its study becomes important. Some important features can be extracted from an edge of any image (e.g.: corners, lines, curves).

\section{REVIEW OF LITRATURE}

Zolqernine Othman et al., [8] author discussed, that Canny method can produce equally good edge with the smooth continuous pixels and thin edge. Sobel edge detection method cannot produce smooth and thin edge compared to canny method. But same like other method, Sobel and Canny methods also very sensitive to the noise pixels. Sometime all the noisy image cannot be filtered perfectly. Unremoved noisy pixels will affect the result of edge detection. From the analysis, it was shown that between Sobel and Canny edge detection algorithms, response given by Canny edge detection was better than result of Sobel detector used in these MRI images.

M Sudarshan et al., [9] author proposed an optimized edge detection algorithm suitable for the face recognition task. The main idea of the proposed method is to boost the significant edges and then apply successive thinning algorithms. The two advantages of this method over other gradient based systems is its ability to find missing and broken edges more accurately and suppress the less significant edges. Possible future work is to enhance the thinning algorithm such that it is able to suppress the noise in post processing stage more effectively.

C.NagaRaju et.al (2011)[10] proposed a novel edge detection algorithm based on multi-structure elements morphology of eight different directions. Here, the eight different edge detection results are obtained by using morphological gradient algorithm respectively, and then the final edge results are obtained by using synthetic weighted method. The proposed algorithm is more efficient for edge detection than conventional mathematical morphological edge detection algorithms and differential edge detection operators

W. Luo [6] suggested that images are often corrupted by noise known as salt and pepper noise. This noise can corrupt the images and the corrupted pixel takes either maximum or minimum gray level. Along with these standard median filters has been established as reliable method to remove the salt and pepper noise without harming the edge features. Though, the major problem of standard Median Filter (MF) is that the filter is effective only at low noise densities.

PriyankaKamboj and Versha Rani [7] have studied various noise model and filtering techniques. In image processing, noise reduction and image restoration is expected to improve the qualitative inspection of an image and the performance criteria of quantitative image analysis techniques. Digital image is inclined to a variety of noise which affects the eminence of image. The purpose of image denoising is to restore the detail of original image to the great extent. The criterion of the noise removal problem depends on the type of noise by which is corrupting the image. Different methods for reduction of noise and image enhancement have been considered.

Yong Wang et al. (2012) [19] has done the comparative study of research work done in the field of image filtering. Image filtering processes are applied on images to remove the different types of noise that are either present in the image during capturing or introduced into the image during transmission. The salt \& pepper(impulse) noise is the one type of noise which is occurred during transmission of the images or due to bit errors or dead pixels in the image contents. The images are blurred due to object movement or camera displacement when we capture the image. This pepper deals with removing the impulse noise and blurredness simultaneously from the images. The hybrid filter is a combination of wiener filter and median filter.

K. Sai Deepak et.al (2012)[11] proposed a two-stage methodology for the detection and classification of Diabetic macularedema (DME) severity from color fundus images. DME detection is carried out via a supervised learning approach using the normal fundus images. The feature extraction technique is introduced to capture the global characteristics of the fundus images and discriminate the normal from these images. Disease severity is assessed using a rotational asymmetry metric by examining the symmetry of macular region.

HosseinGhayoumiZadeh et. al (2013)[12] proposed an image analysis approach for automated detection and classification of particular cells, specially the cancer cell from normal cells. In this technique, we can also count the number of defected cells and then also find their position using image processing. The results of this analysis are useable in designing a neural 
network for more accurate analysis. The particular cells segregation is the most important property of this work.

Zhao(2001)[21]proposed an edge detection technique based on probability partition of the image into 3-fuzzy partitions (regions) and the principle of maximum entropy for finding the parameters value that result in the best compact edge representation of images.

\section{ACKNOWLEDGMENTS}

The authors are highly grateful and thankful to the Dr. Baljit Singh Khehra(Head Of CSE \& IT Department) of the Baba Banda Singh Bahadur Engineering College, Fatehgarh Sahib and to the college also.

\section{CONCLUSION}

This paper represents the theoretically based study of image segmentation methods which gives insights of mostly used edge detection techniques. The mostly edge detection techniques are gradient based i.e. Robert, sobel, prewitt operator and laplacian based laplacian of Gaussian and canny edge detection. An adaptive edge detection algorithm is necessary to give a rough and tough solution that is adaptable to the varying noise levels. The gradient based method such as prewitt filter has the most important drawback of being sensitive to the noise. Canny edge detection is less sensitive to the noise but is more expensive than Robert, Sobel, and prewitt operator. However, canny edge detection performs better than all these operators in anyway.

\section{REFERENCES}

[1] W. Frei and C. Chen, "Fast Boundary Detection: A Generalization and New Algorithm," IEEE Trans. Computers, vol. C-26, no. 10, pp. 988-998, Oct. 1977.

[2] J. Canny, "A computational approach to edge detection," IEEE Trans. Pattern Analysis and Machine Intelligence, Vol. 8, No. 6, pp. 679-698, Nov. 1986

[3] Raman Maini and Dr. Himanshu Aggarwal "Study and Comparison of various Image Edge Detection Techniques" International Journal of Image Processing (IJIP), Vol3: Issue (1).

[4] S.Lakshmi and Dr.V.Sankaranarayanan "A study of Edge Detection Techniques for Segmentation Computing Approaches" IJCA Special Issue on Imaging and Biomedical Applications" CASCT, 2010. [3] Edge Detection by Trucco,Chapter 4 and Jain ctal.,Chapter 5.

[5] Raman Maini and Dr. Himanshu Aggarwal "Study and Comparison of various Image Edge Detection Techniques" International Journal of Image Processing (IJIP), Vol3: Issue (1).

[6] W.Luo, Efficient Removal of Impulse Noise from Digital Images”, IEEE Transactions, 2006, 523527.

[7] P.Kamboj, V.Rani, Image Enhancement Using Hybrid Filtering Technique, IJSR, vol. 2(6), 2013, 214-220.
[8] Zolqemine Othman, habibollahharon, Mohammed Rafi, Abdul kadir, -Comparison of canny and Sobel edge detection in mri images.

[9] M sudarsha*" $p$ ganga Mohan and suryakanth v gangashetty -Optimized edge detection algorithm for face recognition".

[10] C.NagaRaju ,S.NagaMani, G.rakesh Prasad, S.Sunitha,"Morphological Edge Detection Algorithm based on Multi-Structure Elements of Different Directions",IJICT, Volume 1 No. 1, May 2011.

[11] K Sai Deepak JayanthiSivaswamy,"Automatic Assessment of Macular Edema from ColorRetinalImages",IEEE TRANSACTIONS ON MEDICAL IMAGING, VOL. 31, NO. 3 MARCH 2012.

[12] HosseinGhayoumiZadeh, SiamakJanianpour, and JavadHaddadnia,"Recognition and Classification of Cancer cells by using Image Processing and LABVIEW", International Journal of Computer Theory and Engineering, Vol. 5, No. 1, February 2013.

[13] R. C. Gonzalez and R. E. Woods, Digital Image Processing. Upper Saddle River, NJ: Prentice-Hall, 2001, pp. $572-585$

[14] W. K. Pratt, Digital Image Processing. New York, NY: Wiley-Interscience, 1991, pp. 491-556.

[15] 3J. Canny, "Finding Edges and Lines in Images," Massachusetts Institute of Technology, Cambridge, Massachusetts, USA, Tech. Rep. no. 720, 1983

[16] B.Goossens.Q.LuongA.Pizurica.W Phillips, An improved non-local denoising algorithm, in: Local and Non-Local Approximation in Image Processing International Workshop, Proceeding,2008,pp.143-156.

[17] G.W. Wei, Discrete singular convolution for the solution of the Fokker-Planck equations, J. Chem. Phys. 110 (1999) 8930-8942.

[18] R.H.Chan, C.W. Ho,andM.Nikolova, "Salt-and-Pepper Noise Removal by Median-Type Noise Detectors and Detail-Preserving Regularization," IEEE Transactions on Image Processing, vol. 14, No. 10, pp.1479-1485, 2005.

[19] Wang, Changhong, T. Chen, and Z. Qu., "A novel improved median filter for salt and pepper noise from highly corrupted images." In Systems and Control in Aeronautics and Astronautics (ISSCAA), vol. 3, pp. 718 722, 2010.

[20] Daniel L. Schmoldt, Pei Li and A. Lynn Abbott, "Machine vision using artificial neural networks with local 3D neighbourhoods", Computers and Electronics in Agriculture, vol.16, 1997, pp.255-271.

[21] M. Zhao, A. Fu, and H. Yan, "A Technique of ThreeLevel Thresholding Based on Probability Partition a Fuzzy 3-Partition,"IEEE Trans. on Fuzzy Systems, vol.9, no.3, pp. 469 479, June 2001. 